\title{
Development of a Magnetic Cusp Gun for a 1-THz Harmonic Gyrotron
}

\author{
Fan-Hong Li, Chao-Hai Du, Senior Member, IEEE, Zi-Wen Zhang, Si-Qi Li, Zi-Chao Gao, \\ Juan-Feng Zhu, Guo-Wu Ma, Qi-Li Huang, Hong-Ge Ma, Liang Zhang, Senior Member, IEEE, \\ Adrian W. Cross, Member, IEEE, Pu-Kun Liu, Senior Member, IEEE
}

\begin{abstract}
High-quality axis-encircling electron beam is essential to drive a high-harmonic gyrotron. A magnetic cusp gun that can generate a low-spread axis-encircling electron beam at an extremely high magnetic-compression ratio was developed for a 1-THz 4th-harmonic gyrotron toward continuous-wave operation. The optimized gun could generate an axis-encircling electron beam with $80-\mathrm{kV}$ voltage, 1-A current, and a nearly constant pitch factor of about 1.5 in a wide range of magnetic field strength. The transverse velocity spread was less than $2.1 \%$ and the guiding center radius was $26.6 \mu \mathrm{m}$. The design was verified by using MAGIC, EGUN, and CST simulations and good agreements were achieved.
\end{abstract}

Index Terms-Magnetic cusp gun, axis-encircling beam, THz gyrotron

\section{INTRODUCTION}

$\mathrm{G}$ YROTRONS based on the electron cyclotron maser, are capable of effectively generating electromagnetic (EM) radiation [1]-[3]. According to the synchronization condition between the operating mode and electrons, the radiation frequency can be approximated as

$$
\omega \approx s e B_{z i} / \gamma m_{0}
$$

where $\omega$ is the angular frequency, $e$ is the electron charge, $m_{0}$ is the electron rest mass, $B_{z i}$ is the magnetic field strength, $s$ is the harmonic number, and $\gamma$ is the relativistic factor. According to (1), generating high-frequency radiation requires strong magnetic fields. To effectively reduce the required magnetic field strength, high-harmonic beam-wave interaction is employed, since the magnetic field strength is inversely proportional to $s$. However, high-harmonic beam-wave interaction still encounters mode competition between the operating waveguide mode and parasitic modes. Using the

This work was supported by the National Science Foundation of China under Contract 61531002, Contract NSAF U1830201, and Contract 61861130367 . This work was also supported in part by Newton Advanced Fellowship NAF\R1\180121 from Royal Society, United Kingdom.

F. H. Li, C. H. Du, Z. C. Gao, Z. W. Zhang, J. F. Zhu, S. Q. Li and P. K. Liu are with the department of electronics, Peking University, Beijing 100871, China (e-mail: duchaohai@pku.edu.cn).

G. W. Ma, Q. L. Huang, and H. G. Ma are with the Institute of applied electronics, China Academy of Engineering Physics, Mianyan 621900, Sichuan, China.

A. W. Cross, and L. Zhang are with the Department of Physics, SUPA, University of Strathclyde, Glasgow G4 0NG, United Kingdom. axis-encircling electron beam, in other words, the large orbit beam (LOB), could be an option to suppress the mode competition. The mode selectivity of LOBs can be explained by the beam-wave coupling factor [3]

$$
H_{s m}=J_{s-m}^{2}\left(k_{m n} r_{g}\right) J_{s}^{\prime 2}\left(k_{m n} r_{L}\right)
$$

where $J$ is the Bessel function of the first kind, $k_{m n}$ is the $\mathrm{TE}_{\mathrm{m}, \mathrm{n}}$ mode cut-off wavenumber, and $r_{g}, r_{L}$ is the guiding center radius and the Larmor radius of the electrons, respectively. For an ideal LOB whose guiding center radius is 0 , those waveguide modes with $\mathrm{s} \neq \mathrm{m}$ cannot couple with the electrons, therefore eliminating this group of parasitic modes.

Generating LOBs for gyrotrons has been explored in the past decades [4]-[11]. According to the different configurations of the magnet systems, the approaches to generate LOBs can be divided into three types. The first is to add a kicker coil into the main magnet system. It provides an additional transverse force to the electrons[6]. However, it is challenging to realize such a non-symmetrical magnetic field [12], [13]. The reported velocity spread was relatively high and not good enough[7], [8], [14]-[16]. The second way to generate LOBs is to adopt a magnet system containing a sharp magnetic field cusp region provided by magnet poles [17]-[20]. The electron guns using this type of magnet system are usually called Magnetic Cusp Guns (MCGs). The third way is an improvement of the second one, in which a smooth magnetic cusp is adopted instead of the ideal cusp. The reversed magnetic field is provided by auxiliary axisymmetric coils instead of magnet poles. The third approach has a simpler configuration of the magnet system compared with the first two approaches. Although it is relatively convenient to deploy such a smooth cusp magnet system, it is still challenging to generate a low velocity-spread electron beam at an ultra-high magnetic compression ratio.

An MCG with a smooth cusp system has been designed based on the experience of the existing work in this paper, and the capacity for magnetic tunning has been investigated as well. The paper is organized as follows. The design and optimization of the MCG are detailed in Section II. The influence of surface roughness and cathode temperature is discussed in Section III. The conclusions are presented in Section IV.

\section{Design AND ANALYSIS OF the LARGE ORBIT GuN}

\section{A. Theory of Electron Dynamics}

Lagrange mechanics are used to analyze the motion of electrons. The Lagrangian for the dynamics of the electrons in the large orbit gun with axisymmetric electrostatic and 
magnetostatic fields can be written as [17]

$$
L=-m_{0} c^{2}\left[1-\left(\dot{r}^{2}+r^{2} \dot{\theta}^{2}+\dot{z}^{2}\right) / c^{2}\right]+e \phi-e r \dot{\theta} A_{\theta}
$$

where $\phi$ is the scalar potential. $r, \theta, z$ are the three coordinates in the cylindrical system, and $A_{\theta}$ is the magnetic vector potential. $A_{\theta}$ could be represented as [17]

$$
A_{\theta}=r B_{z} / 2
$$

According to the Euler-Lagrange equation, the Lagrangian in azimuthal coordinate yields

$$
\frac{d}{d t}\left(\frac{\partial L}{\partial \dot{\theta}}\right)=\frac{\partial L}{\partial \theta}
$$

The left-hand side is the derivative of generalized angular momentum (GAM) to time $t$, which is

$$
P_{\theta}=\frac{\partial L}{\partial \dot{\theta}}=m_{0} \gamma r^{2} \dot{\theta}-e r A_{\theta}
$$

In (3), the first term on the right-hand side does not explicitly contain $\theta . \phi$ and $A_{\theta}$ are the potentials of the axisymmetric static field. Thus, the derivative of $L$ to $\theta$ in (5) is 0 . Therefore, the GAM keeps constant, which is normally called the conservation of GAM. Assuming that the angular velocity $\dot{\theta}$ is zero on the emitter surface, the GAM could be derived as

$$
P_{\theta c}=-e r_{c}^{2} B_{z c} / 2
$$

where $r_{c}$ represents the radius of the emitter center. The GAM at the beam-wave interaction cavity could be written as

$$
P_{\theta i}=-e B_{z i}\left(r_{g}^{2}-r_{L}^{2}\right) / 2
$$

where $r_{g}$ is the guiding center radius of the electron beam, and $r_{L}$ is the Larmor radius of the gyrating electrons. For a magnetic field of $B_{z i}, r_{L}$ can be expressed as

$$
r_{L}=\frac{\gamma m_{0} v_{t}}{e B_{z i}}
$$

Based on the (7)-(9), two important parameters can be derived. Firstly, the pitch factor $\alpha$, which is defined by the ratio of transverse velocity to the axial velocity, can be derived as

$$
\alpha=\left[\frac{\left(\gamma^{2}-1\right) c^{2} m_{0}^{2}}{B_{z i}\left(B_{z i} r_{g}^{2}-B_{z c} r_{c}^{2}\right) e^{2}}-1\right]^{-1 / 2}
$$

This equation can be used to determine the initial parameters of the emitter. Secondly, the transverse velocity spread as a function of $\Delta P_{\phi c}, \Delta v_{t}$, and $\Delta r_{g}$ can be expressed as

$$
\frac{\Delta v_{t}}{v_{t}}=\frac{r_{L}^{2}-r_{g}^{2}}{r_{L}^{2}} \cdot \frac{\Delta P_{\phi c}}{2 P_{\phi c}}+\frac{r_{g}^{2}}{r_{L}^{2}} \cdot \frac{\Delta r_{g}}{r_{g}}
$$

This equation shows the possibility to reduce the velocity spread by adjusting the spread of the guiding center and the initial GAM. For the non-ideal cusp model with accelerated electrons, the following equation can be derived from the Lagrange equation of the radial component [17], [19]

$$
\dot{r}=\frac{\omega_{c} \zeta}{2} \sin ^{-1} \frac{1}{\eta}+v_{r 0}
$$

where $\zeta$ is a scale factor, $\omega_{c}$ is the electron cyclotron frequency, $\eta=v_{0} / r_{0} \omega_{c}, v_{0}$ is the injection beam velocity and $r_{0}$ is the injection beam radius. According to (12), $r_{g}$ is induced from the non-ideal magnetic field profile and the radial velocity of electrons before the cusp region. $r_{g}$ can be minimized through the optimization of the magnetic field profile through the magnet system and the electric field through the geometries of the cathode and the anode.

\section{B. Design and Optimization of the MCG}

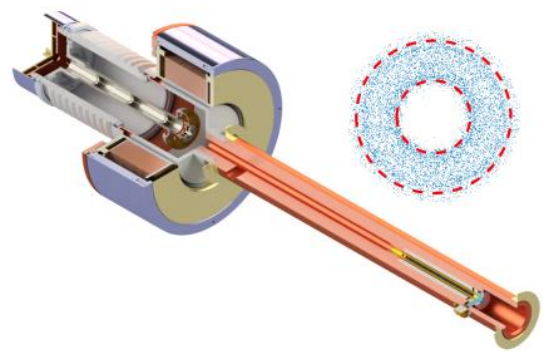

Fig. 13-D view of the total MCG. The inset is the cross-view of the beam trajectories in the interaction cavity. The radius of the red inner circle is $r_{L}-r_{g}$, while the radius of the red outer circle is $r_{L}+r_{g}$.

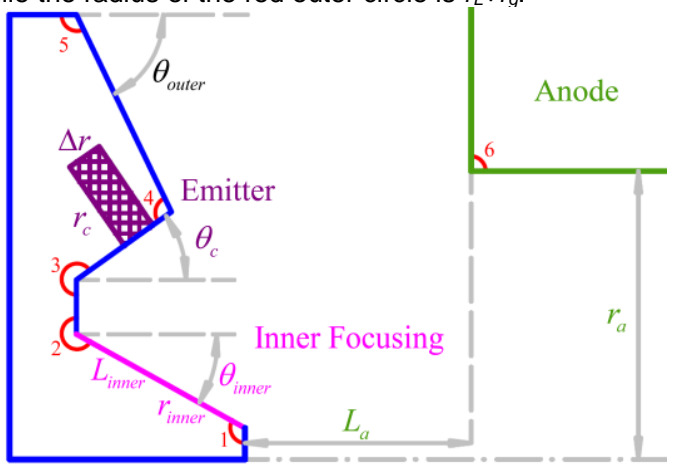

Fig. 2. Design Scheme of the cathode and the anode (green). The cathode can be divided into three parts, i.e., the emitter (purple), the inner focusing electrode (magenta), and the outer focusing electrode. For showing the label of the related parameters, the scale in this figure is not precisely in agreement with the values in Table I. Six parts are tagged by red arches. These parts will be changed into round corners to prevent the MCG from breakdown.
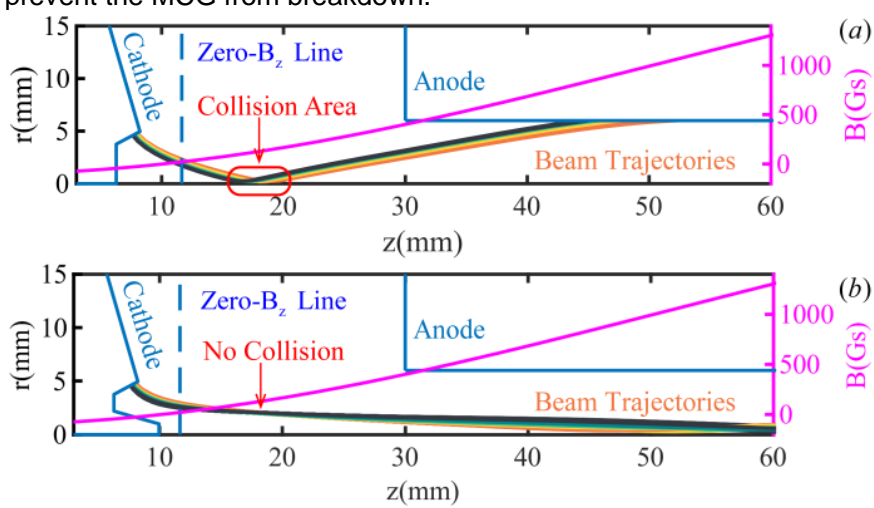

Fig. 3 Trajectories of electron beams in the MCG (a) without the inner focusing electrode and (b) with the inner focus.

TABLE. I

TARget Values Related to the Performance of the MCG

\begin{tabular}{lll}
\hline \hline Parameters & Symbol & Value \\
\hline Pitch Factor & $\alpha$ & $\sim 1.5$ \\
Main Magnetic Field & $B_{z i}$ & $9.0 \mathrm{~T}$ \\
Emission Current & $I$ & $1 \mathrm{~A}$ \\
Acceleration Voltage & $U$ & $80 \mathrm{kV}$ \\
\hline \hline
\end{tabular}

The key parameters of the MCG shown in Table I are determined by the beam-wave interaction of the $1-\mathrm{THz}$ gyrotron. As the gyrotron has relatively low beam-wave coupling in the harmonic interaction, a large current, high pitch 


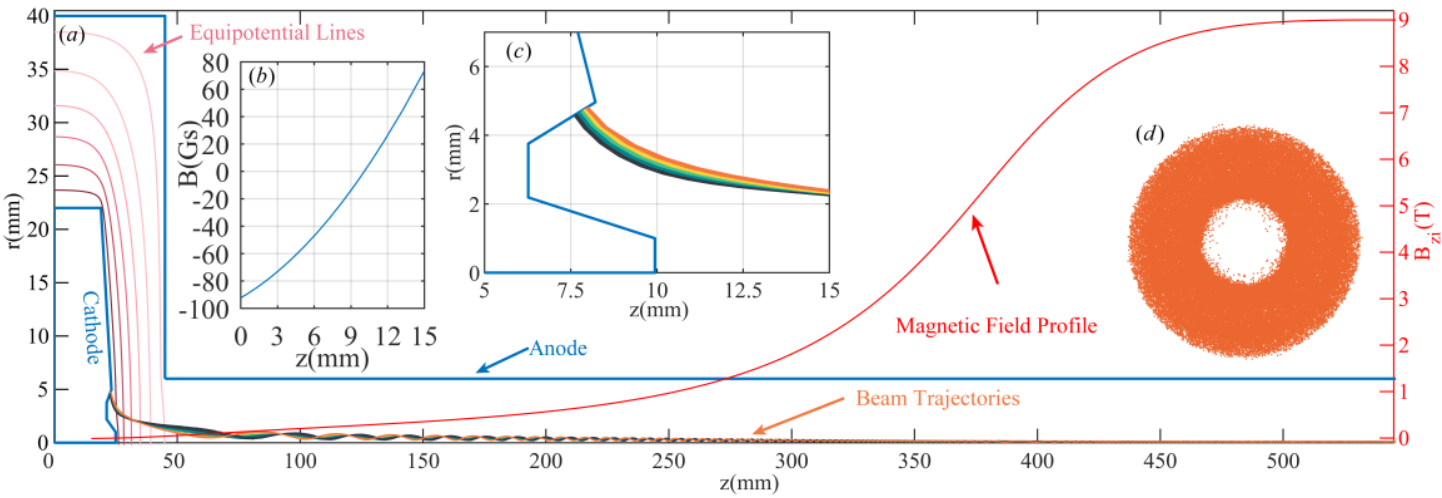

Fig. 4. Trajectories of the LOBs. (a) the magnetic field profile near the cathode(b) 2-D view of the MCG and simulated trajectories. (c) Details of the emitter and the inner focusing electrode. (d) Cross-section view of the generated beams.

factor and low velocity spread electron beam is desired to achieve larger output power. These are also the design targets of the MCG presented in this paper. The structure of the cathode and anode is derived from the pierce guns [21], [22]. The design diagram is shown in Fig. 1 and Fig. 2. To reduce the complexity in modeling, the round corners in the MCG are ignored firstly in the optimization stage, however, they will be included in the final simulations.

Firstly, the emitter contains four parameters, including the oblique angle $\theta_{c}$, the emission width $\Delta r$, the mean radius $r_{c}$, and the magnetic field strength at the emission surface $B_{c}$. The initial values of $\theta_{c}, \Delta r, r_{c}$, and $B_{c}$ are derived based on (7)-(10). $r_{g}$ is set as 0 to estimate the initial parameters. $r_{c}$ and $B_{c}$ are calculated based on (10). The initial values of $r_{c}$ and $B_{c}$ are 5.0 $\mathrm{mm}$ and $\sim 30 \mathrm{Gs}$, respectively. The compression ratio is $\sim 3000$. $\theta_{c}$ is related to the profile of the magnetic field. A small velocity spread is required to keep a conformal relationship between the profile of the magnetic field and the emitter [10]. It should be noted that the profile of the magnetic field at the emitter region is normally not linear, which means the emitter should also be curved. However, a curved emitter is challenging to anufacture therefore the emitter in this paper is a straight line in the RZ plane. The slope of the straight line is depicted by $\theta_{c}$. Different oblique angles will result in three kinds of beam flows, i.e., the symmetrical cross-flow, the positive cross flow, and the negative cross-flow [10]. The symmetrical cross-flow is chosen as the initial design, and the corresponding $\theta_{c}$ is $38^{\circ}$. $\Delta R$ is set as $0.5 \mathrm{~mm}$, empirically. The corresponding emission current density is $6.77 \mathrm{~A} / \mathrm{cm}^{2}$ for a 1-A emission current.

Secondly, the focusing electrode has three parameters, i.e., the oblique angle $\theta_{\text {inner }}$, the length $L_{\text {inner }}$, and the radius $r_{\text {inner }}$. Due to the small radius and oblique angle of the emitter, the electrons emitted from the surface tend to travel to the symmetric axis of the MCG. The large radial velocity will lead the electrons to strike on the anode wall, as shown in Fig. 3 (a). The collisions can be effectively reduced with the employment of the inner focusing electrode. The effect of the inner focusing electrode can be visualized in Fig. 3 (a) and (b). It has the same potential as the cathode. The electric field at the emitter region will be reshaped to reduce the radial velocities of the electrons. The emitted electron can therefore smoothly transit into an axis encircling beam as shown in Fig. 3(b). With the inner focusing electrode, the electron beam is compressed in the concave region before the zero- $B_{z}$ point, which helps to suppress the velocity spread [19]. The inner focusing electrode should match with the emitter. When the emitter has large $\theta_{c}$ and $r_{c}$, the collision is not likely to occur, and the inner focusing electrode can even be removed, for example in the design in Refs. [9], [13], [23], [24].

Thirdly, the anode is also an essential part of an electron gun. Normally, there is a tip structure in the anode, and in the past decades, this sort of structures or similar designs were widely employed [10], [12]-[15], [19], [23]-[28], and some of them were verified experimentally. Since both the anode tip and the inner focusing electrode provide centrifugal forces with opposite directions, it is possible to simplify the anode tip structure as shown in Fig. 2. It also benefits to reduce the number of parameters needed to be optimized and the computing time for optimization. Only two parameters are needed to construct the anode. i.e., the inner radius $r_{a}$ and the distance $L_{a}$ between the anode and the cathode. To avoid breakdown between the cathode and the anode, $L_{a}$ should be large enough. The limit for the electric field strength to avoid breakdown is $10 \mathrm{kV} / \mathrm{mm}$ in this manuscript. Also, in the magnetic field compression region, the average radius of electrons gradually reduces. Therefore, the initial value of $r_{a}$ can be slightly larger than $r_{c}$, which can effectively prevent the electrons from hitting the anode wall in the simulations with initial values. The MCG with these initial geometry parameters was simulated. The average of the transverse velocity spread $\delta v_{t}$, the pitch factor $\alpha$, and the guiding center radius $r_{g}$ were calculated from the electron trajectories to evaluate the beam quality. Further optimization was carried out to find the minimum of the average $\delta v_{t}$ and to make $r_{g}<0.2 \lambda$, where $\lambda$ is the operating wavelength [9]. In the optimization, $B_{z c}$ is adjusted to keep the average $\alpha$ to 1.5. By sweeping parameters around the initial values, the optimized value of the emitter parameters was found, as shown in Table II. The LOBs generated from the optimized MCG are shown in Fig. 4. The thickness of the electron beams shown in Fig. 1 and Fig. 4 is induced by the spread of $r_{L}$ and nonvanishing $r_{g}$. Since $r_{L}$ is $\sim 4$ times higher than $r_{g}$, the thickness looks large visually. In the optimization, it is not necessary to minimize $r_{g}$, because the proper value and distribution of $r_{g}$ will help to lower the velocity spread according to (11).

\section{Simulation Results and Analysis about the Design of the MCG.}

The beam quality under various voltages and currents are shown in Fig. 5 and 6, respectively. In the range from $1 \mathrm{~A}$ to $2 \mathrm{~A}$, 


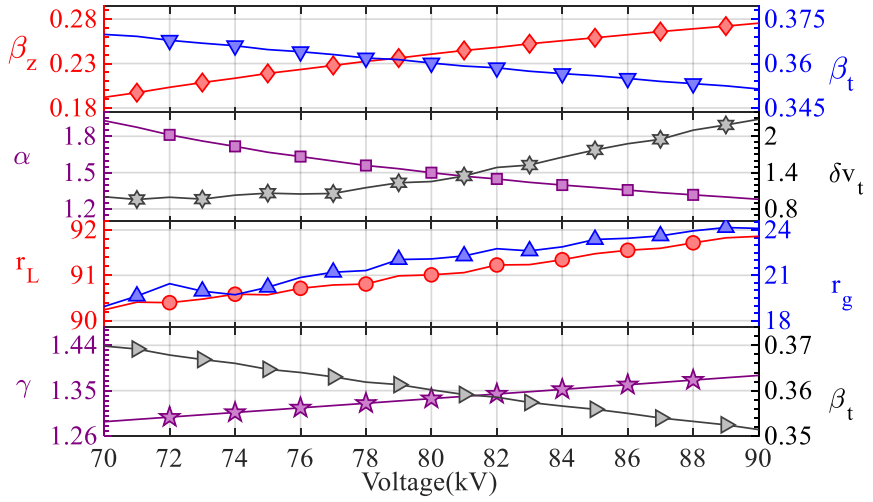

Fig. 5 Pitch factors, normalized transverse velocities $\beta_{t}=v_{t} / \mathrm{c}$, normalized axial velocities $\beta_{z}=v_{z} / c$ and the spread of transverse velocities $\delta v_{t}$. The unit of $r_{L}$ and $r_{g}$ is $\mu \mathrm{m}$. The unit of $\delta v_{t}$ is \%.

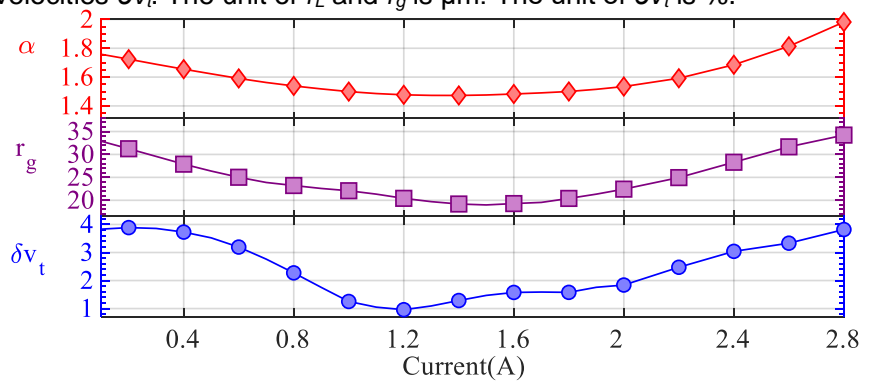

Fig. 6. Beam quality parameters under various emission currents. The unit of $r_{L}$ and $r_{g}$ is $\mu \mathrm{m}$. The unit of $\delta v_{t}$ is $\%$.
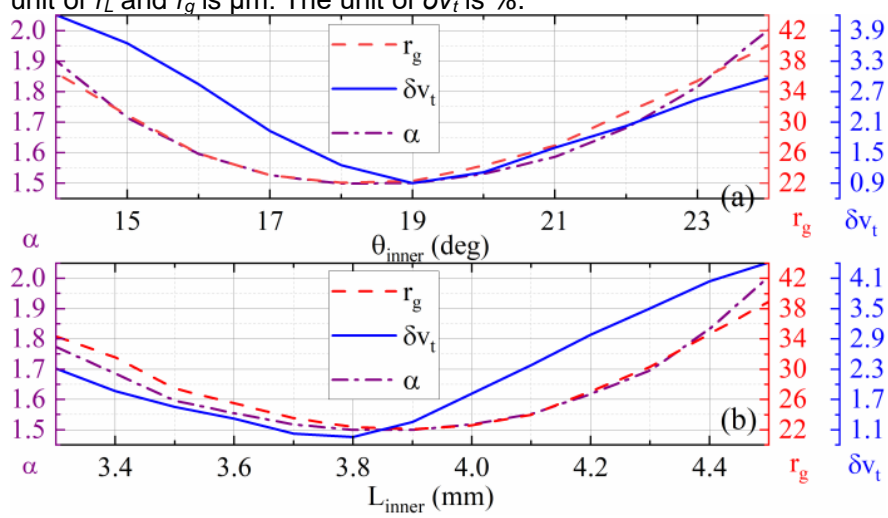

Fig. 7 Beam quality parameter under various (a) oblique angles and (b) lengths of the inner focusing electrode. The unit of $r_{g}$ is $\mu \mathrm{m}$. The unit of $\delta v_{t}$ is \%.

the geometry can effectively suppress the space charge effect. Therefore, the velocity spread can be lower than $2 \%$. In both the low and the high emission-current range, the poor suppression of the space charge effect induces the increase of the velocity spread. When the emission current is larger than $2.6 \mathrm{~A}$, part of the emission electrons is reflected. The number of the reflected electrons becomes larger with the increase of the emission current. The thermionic cathodes for the $\mathrm{THz}$ gyrotron will operate in the temperature-limited emission regime [29]. In this operating regime, the emission current is mainly governed by the temperature of the emitter [30]. The optimal operating current is around 1.2 A, the beam quality is not sensitive to the current value. From Fig. 5, $r_{g}$ becomes larger with the increment of the operating voltage, while from Fig. 6, $r_{g}$ decreases with the increment of the operating current. Based on the analysis of (12), the dissimilarity between them is induced by the change in acceleration field and space charge field. The acceleration field provides a centripetal force on electrons, while the space-charge field provides a centrifugal

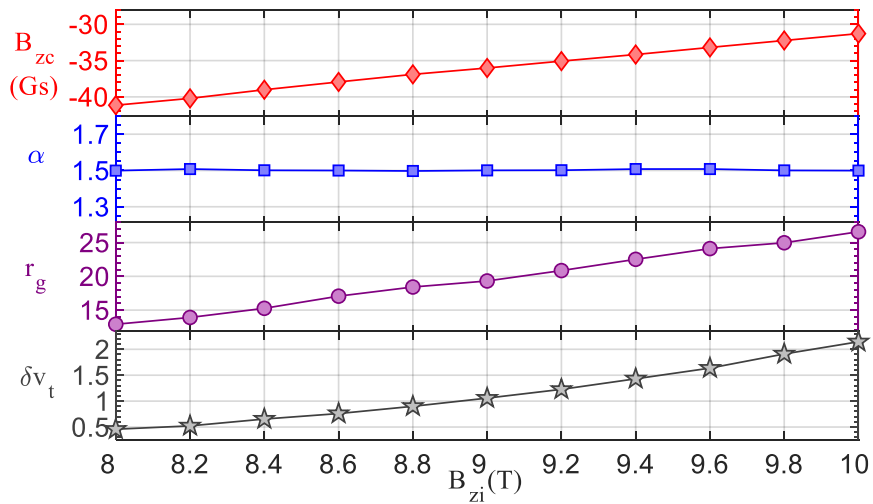

Fig. 8. Beam parameters under various $B_{z i}$. The unit of $r_{g}$ is $\mu \mathrm{m}$. The unit of $\delta v_{t}$ is \%.

TABLE III

RADIUSES OF THE ROUNDS CORNERS IN THE MCG

\begin{tabular}{llllll}
\hline \hline & $B_{z c}(\mathrm{G})$ & $\alpha$ & $r_{L}(\mu \mathrm{m})$ & $r_{g}(\mu \mathrm{m})$ & $\delta v_{t}(\%)$ \\
\hline \hline MAGIC & -35.23 & 1.53 & 91.01 & 22.08 & 1.25 \\
EGUN & -31.05 & 1.49 & 90.66 & 23.58 & 1.27 \\
CST & -31.62 & 1.50 & 91.37 & 32.48 & 3.40 \\
\hline \hline
\end{tabular}

force on electrons. The change of the operating voltage alters the acceleration field, which increases the centripetal acceleration, and the change of emission current alters the space-charge field which increases the centrifugal acceleration. That is the reason why $r_{g}$ differs when tuning the emission current and acceleration voltage. The parameters for the inner focusing electrode were investigated. The performance under various $\theta_{\text {inner }}$ and $L_{\text {inner }}$ is shown in Fig. 7. The profiles of $\alpha, r_{g}$, and $\delta v_{t}$ vs. $r_{\text {inner }}$ are similar to those under $\theta_{\text {inner }}$ and $L_{\text {inner. }}$. Therefore, they are omitted here. The beam quality parameters $\alpha, r_{g}$ and $\delta v_{t}$ are sensitive to $\theta_{\text {inner }}$ and $L_{\text {inner }}$ and $r_{\text {inner }}$, which means a tight tolerance is required in manufacture. The performance for magnetic tuning is shown in Fig. 8. $B_{z c}$ increases linearly with the increment of $B_{z i}$. When $\alpha$ and $U$ keep invariant, $r_{L}$ will decrease gradually according to (10). $r_{g}$ will increase monotonically. The values of $r_{g}$ at $8 \mathrm{~T}$ and $10 \mathrm{~T}$ are $12.9 \mu \mathrm{m}$ and $26.6 \mu \mathrm{m}$, respectively, which is $4.30 \%$ and $8.87 \%$ of the wavelength at $1 \mathrm{THz}$. which is acceptable [9]. The maximum $\delta v_{t}$ is $2.15 \%$. To further verify the performance of the MCG, the design model was simulated by EGUN [31], MAGIC and CST Particle Studio. The simulation results are shown in Table III.

\section{Round corners in the MCG}

TABLE. IV

RADIUSES OF THE BEND IN THE MCG

\begin{tabular}{ll}
\hline \hline Cathode round corners & Radius $(\mathrm{mm})$ \\
\hline \hline 1 & 0.85 \\
2 & 0.9 \\
3 & 0.9 \\
4 & 0.3 \\
5 & 8.0 \\
Anode bend & Radius $(\mathrm{mm})$ \\
6 & 2.0 \\
\hline \hline
\end{tabular}

The six round corners are shown in Fig. 2. The radii of them are shown in Table IV. The simulation model was established and calculated using CST due to the convenience of blend operations. The simulation results on the beam quality are shown in Table V. and VI. 
TABLE. $V$

THE SIMULATION RESULTS WITH AND WITHOUT ROUND CORNERS

\begin{tabular}{lllll}
\hline \hline & $\alpha$ & $r_{L}(\mu \mathrm{m})$ & $r_{g}(\mu \mathrm{m})$ & $\delta v_{t}$ \\
\hline \hline With round corners & 1.671 & 94.02 & 38.19 & $4.9 \%$ \\
Without round corners & 1.498 & 91.37 & 32.40 & $3.4 \%$ \\
\hline \hline \multicolumn{5}{c}{ TABLE. VI }
\end{tabular}

THE ELECTRIC FIELD STRENGTH BEFORE AND AFTER EMPLOYING THE ROUND CORNERS

\begin{tabular}{lll}
\hline \hline Corners & Before $(\mathrm{kV} / \mathrm{mm})$ & After $(\mathrm{kV} / \mathrm{mm})$ \\
\hline \hline 1 & 16.48 & 9.68 \\
2 & 7.05 & 6.28 \\
3 & 9.24 & 5.86 \\
4 & 10.32 & 9.52 \\
5 & 15.99 & 9.15 \\
6 & 2.36 & 2.03 \\
\hline \hline
\end{tabular}

\section{DISCUSSION}

\section{A. Influences of Roughness and Thermal effects of the emitter surface.}

TABLE. VII

The Value of Related Parameter @ 9.0 T, $80 \mathrm{KV}$

\begin{tabular}{llll}
\hline \hline Parameters & Value & Parameters & Value \\
\hline \hline$E_{c}($ average $)$ & $3.7 \mathrm{kV} / \mathrm{mm}$ & $v_{\perp}$ & $1.25 \times 10^{8} \mathrm{~m} / \mathrm{s}$ \\
$F_{m}$ & 2307.7 & $\theta_{c}$ & $32^{\circ}$ \\
$R_{r m s}$ & $112 \mathrm{~nm}(4.4 \mu \mathrm{in})$ & $\left(\delta v_{\perp}\right)_{R}$ & $5.44 \%$ \\
$B_{z c}$ & $-36 \mathrm{Gs}$ & $\left(\delta v_{\perp}\right)_{T}$ & $5.00 \%$ \\
$T_{c}$ & $1300 \mathrm{~K}$ & $\left(\delta v_{\perp}\right)_{\text {total }}$ & $7.49 \%$ \\
\hline \hline
\end{tabular}

The beam quality is influenced by many factors. Some factors included the initial thermal velocity due to the cathode temperature, the roughness and patchiness in the cathode surface, and nonuniform emission are also significant factors on the beam quality [32]. These factors will be discussed in this section. Based on the previous work, these factors were considered mathematically. Most of them are modeled in parallel plate electrodes, which are more suitable for evaluating MIGs than MCGs. Therefore, the related contents are put into the discussion rather than the main body of the paper since it is controversial. Although these models for deriving these equations are not identical with the design model in this paper, it is still useful to provide a baseline for the experiment. The equations are shown as follows [32]-[35].

$$
\begin{gathered}
\left(\delta v_{\perp}\right)_{R}=0.4 \sqrt{\frac{2 e E_{c} R_{r m s} F_{m}}{\gamma m_{0}}} \cdot \frac{1}{v_{\perp}} \\
\left(\delta v_{\perp}\right)_{T}=\sqrt{\frac{k T_{c} F_{m}}{\gamma m_{0}}} \cdot \frac{1}{v_{\perp}}
\end{gathered}
$$

where $E_{c}$ is the electric field strength at the emitter, $R_{r m s}$ is the root mean square radius of the roughness. $F_{m}$ is the compression ratio, which is equal to $-B_{z i} / B_{z c}$, here. $k$ is the Boltzmann constant. $T_{c}$ is the cathode temperature. The values of these parameters and the calculated velocity spread are shown in Table VII. The three types of spread are statistically independent, the overall spread is the square root of the square sum [32]. From the calculation results, it can be found that the velocity spread is very sensitive to the surface roughness due to the high compression ratio.

\section{B. Setup for the Numerical Software in This Manuscript.}

Three numerical software EGUN, MAGIC and CST Particle Studio are used in this paper. In this subsection, the mesh size and convergence are presented in detail. The mesh size in EGUN is $0.0035 \mathrm{~mm}$ in $\mathrm{z}$ - and $\mathrm{r}$ - directions. The mesh size in MAGIC is nonuniform. Before the anode hole, the max size is $0.05 \mathrm{~mm}$ in both directions. After the anode hole, the max size is $1 \mathrm{~mm}$ in both directions. The time step used in the MAGIC simulations is $60.72 \mathrm{fs}$. In emission surface, the mesh size in CST is $0.05 \mathrm{~mm}$ in $\mathrm{x}-, \mathrm{y}$ - and $\mathrm{z}$-directions. Near the cathode it is $0.5 \mathrm{~mm}$ in all directions. In other areas, it is $2 \mathrm{~mm}$ in all directions. In MAGIC 2-D simulations, special treatment will happen automatically when the mesh sizes in both directions match well. Such treatment can effectively improve simulation accuracy. To make the treatment occur in the emission area, we adjusted the mesh size in both directions. Hence, the results from EGUN and MAGIC are very similar although the mesh size between them differs a lot. The difference in the electric field near the emission area brings fluctuations in the particle emission. The fluctuation influence more on the velocity spread than the velocity means of particles. Therefore, $\delta v_{t}$ is quite different between CST and the other two software. According to (12), the guiding center radius can be minimized when the two terms on the right-hand side of (12) match well, which means $r_{g}$ will elevate with both decrease and increase of particle velocity in the optimized gun structure. Because of the space charge effect, the emission position is not exactly at the emission surface, which is usually called the virtual cathode effect. The positions of the visual position are not the same when the E-fields near the emission surface are different, which means that the particles were emitted from different three tools with the consideration of the virtual cathode effect. In MAGIC and EGUN, the axisymmetric boundary condition was adopted in simulations. Regular 200-sided polygons were employed in replace of cylinders in CST simulations to improve the accuracy. This kind of approximate and the differences in the virtual cathode effect are likely to bring deviations for the simulation results as well.

\section{CONCLUSION}

In this paper, an MCG was designed for the generation of low spread electron beams at a high magnetic-compression ratio. Based on the classical Pierce gun configuration, the structure of the MCG is proposed. The design process of the MCG can be significantly simplified. According to the compensation principle based on the conformal cross-flow, the velocity spread of the generated electron beams can be closed to the analytical minimum even under a high magnetic-compression ratio up to 2300 . The MCG is optimized for a $1-\mathrm{THz} 4^{\text {th }}$-harmonic gyrotron. The operating voltage is 80 $\mathrm{kV}$. The emission current is $1 \mathrm{~A}$. The pitch factor is 1.5 . The Larmor radius is $91.01 \mu \mathrm{m}$, the guiding center radius is 22.08 $\mu \mathrm{m}$, which is $24.26 \%$ of the Larmor radius and $7.36 \%$ of the operating wavelength at $1 \mathrm{THz}$. The transverse velocity spread is $1.25 \%$. The design model is validated by different simulation software. The design method employed in this paper is helpful for the generation of LOBs under ultra-high magnetic-compression ratio and the advancement of high-harmonic gyrotrons towards operation at $1 \mathrm{THz}$. 


\section{REFERENCES}

[1]

G. S. Nusinovich, M. K. Thumm, and M. I. Petelin, "The gyrotron at 50: Historical overview," J. Infrared Millim. Terahertz Waves, vol. 35, no. 4, pp. 325-381, 2014. DOI: 10.1007/s10762-014-0050-7.

[2] J. H. Booske, R. J. Dobbs, C. D. Joye, C. L. Kory, G. R. Neil, G.-S. Park, J. Park, and R. J. Temkin, "Vacuum Electronic High Power Terahertz Sources," IEEE Trans. THz Sci. Technol., vol. 1, no. 1, pp. 54-75, 2011. DOI: 10.1109/tthz.2011.2151610.

[3] K. R. Chu, "The electron cyclotron maser," Rev. Mod. Phys., vol. 76, no. 2, pp. 489-540, 2004. DOI: 10.1103/RevModPhys.76.489.

[4] N. Luhmann Jr, and A. Trivelpiece, "Properties of an RF produced magnetically confined nonneutral hot electron plasma," The Physics of Fluids, vol. 21, no. 11, pp. 2038-2049, 1978. DOI: 10.1063/1.862149.

[5] D. B. McDermott, D. S. Furuno, and N. C. Luhmann, "Production of relativistic, rotating electron beams by gyroresonant rf acceleration in a TE111 cavity," J. Appl. Phys., vol. 58, no. 12, pp. 4501-4508, 1985. DOI: $10.1063 / 1.336262$.

[6] S. Cooke, A. Cross, W. He, and A. Phelps, "Experimental operation of a cyclotron autoresonance maser oscillator at the second harmonic," Phys. Rev. Lett., vol. 77, no. 23, pp. 4836, 1996. DOI: 10.1103/PhysRevLett. 77.4836.

[7] G. G. Denisov, V. L. Bratman, A. W. Cross, W. He, A. D. R. Phelps, K. Ronald, S. V. Samsonov, and C. G. Whyte, "Gyrotron Traveling Wave Amplifier with a Helical Interaction Waveguide," Phys. Rev. Lett., vol. 81 , no. 25 , pp. $5680-5683$, 1998. DOI: 10.1103/PhysRevLett.81.5680.

[8] V. L. Bratman, A. W. Cross, G. G. Denisov, W. He, A. D. R. Phelps, K. Ronald, S. V. Samsonov, C. G. Whyte, and A. R. Young, "High-Gain Wide-Band Gyrotron Traveling Wave Amplifier with a Helically Corrugated Waveguide," Phys. Rev. Lett., vol. 84, no. 12, pp. 2746-2749, 2000. DOI: 10.1103/physrevlett.84.2746.

[9] V. L. Bratman, Y. K. Kalynov, and V. N. Manuilov, "Electron-optical system of terahertz gyrotron," J. Commun. Technol. Electron., vol. 56, no. 4, pp. 500-507, 2011. DOI: 10.1134/s1064226911040024.

[10] C. H. Du, X. B. Qi, B. L. Hao, T. H. Chang, and P. K. Liu, "Conformal Cross-Flow Axis-Encircling Electron Beam for Driving $\mathrm{THz}$ Harmonic Gyrotron," IEEE Electron Device Lett., vol. 36, no. 9, pp. 960-962, 2015. DOI: 10.1109/led.2015.2460457.

[11] V. N. Manuilov, S. V. Samsonov, S. V. Mishakin, A. V. Klimov, and K. A. Leshcheva, "Cusp Guns for Helical-Waveguide Gyro-TWTs of a High-Gain High-Power W-Band Amplifier Cascade," J. Infrared Millim. Terahertz Waves, vol. 39, no. 5, pp. 447-455, 2018. DOI: 10.1007/s10762-018-0473-7.

[12] L. Zhang, W. He, C. R. Donaldson, and A. W. Cross, "Investigation on the optimal magnetic field of a cusp electron gun for a W-band gyro-TWA," Phys. Plasmas, vol. 25, no. 5, pp. 053104, 2018. DOI: 10.1063/1.5027070.

[13] V. Bratman, Y. K. Kalynov, and V. Manuilov, "Large-orbit gyrotron operation in the terahertz frequency range," Phys. Rev. Lett., vol. 102, no. 24, pp. 245101, 2009. DOI: 10.1103/PhysRevLett.102.245101.

[14] V. Bratman, Y. K. Kalynov, V. Manuilov, S. J. R. Samsonov, and Q. Electronics, "Submillimeter-wave large-orbit gyrotron," vol. 48, no. 10-11, pp. 731-736, 2005. DOI: 10.1007/s11141-006-0001-9.

[15] V. L. Bratman, A. E. Fedotov, Y. K. Kalynov, V. N. Manuilov, M. M. Ofitserov, S. V. Samsonov, and A. V. Savilov, "Moderately relativistic high-harmonic gyrotrons for millimeter/submillimeter wavelength band," IEEE Trans. Plasma Sci., vol. 27, no. 2, pp. 456-461, 1999. DOI: $10.1109 / 27.772273$.

[16] E. B. Abubakirov, A. N. Denisenko, A. E. Fedotov, A. N. Leontyev, R. M. Rozental, and V. P. Tarakanov, "Electron-optical system for a high-current Ka-band relativistic gyrotron," Phys. Plasmas, vol. 26, no. 3, pp. 033302, 2019. DOI: 10.1063/1.5085483.

[17] M. J. Rhee, and W. W. Destler, "Relativistic electron dynamics in a cusped magnetic field," Phys. Fluids, vol. 17, no. 8, pp. 1574-1581, 1974. DOI: $10.1063 / 1.1694936$.

[18] W. Lawson, "Design of low velocity - spread cusp guns for axis encircling beams," Appl. Phys. Lett., vol. 50, no. 21, pp. 1477-1479, 1987. DOI: $10.1063 / 1.97805$.

[19] J. Wang, Y. Luo, and N. C. Luhmann, "The simulation of a high-power low-velocity-spread space-charge-limited (SCL) cusp gun," IEEE Trans. Plasma Sci., vol. 38, no. 12, pp. 3356-3362, 2010. DOI: 10.1109/TPS.2010.2085090.

[20] S. G. Jeon, C. W. Baik, D. H. Kim, G. S. Park, N. Sato, and K. Yokoo, "Study on velocity spread for axis-encircling electron beams generated by single magnetic cusp," Appl. Phys. Lett., vol. 80, no. 20, pp. 3703-3705, 2002. DOI: 10.1063/1.1480468.

[21] J. R. M. Vaughan, "Synthesis of the Pierce gun," IEEE Trans. Electron Devices, vol. 28 , no. 1, pp. 37-41, 1981. DOI: 10.1109/T-ED.1981.20279.

[22] J. R. M. Vaughan, "Synthesis of a hollow-beam pierce gun," IEEE Trans. Electron Devices, vol. 34, no. 2, pp. 468-472, 1987. DOI: 10.1109/T-ED.1987.22948.

[23] C. H. Tsai, T. H. Chang, Y. Yamaguchi, and T. Idehara, "Nonadiabatic Effects on Beam-Quality Parameters for Frequency-Tunable Gyrotrons," IEEE Trans. Electron Devices, vol. 67, no. 1, pp. 341-346, 2020. DOI: 10.1109/TED.2019.2954362.

[24] S. Sabchevski, T. Idehara, I. Ogawa, M. Glyavin, S. Mitsudo, K. Ohashi, and H. Kobayashi, "Computer simulation of axis-encircling beams generated by an electron gun with a permanent magnet system," Int. J. Infrared Millim. Waves, vol. 21, no. 8, pp. 1191-1209, 2000. DOI: Doi 10.1023/A:1026491815170.

[25] C. H. Du, T. H. Chang, P. K. Liu, C. P. Yuan, S. J. Yu, G. F. Liu, V. L. Bratman, M. Y. Glyavin, and Y. K. Kalynov, "Development of a Magnetic Cusp Gun for Terahertz Harmonic Gyrodevices," IEEE Trans. Electron Devices, vol. 59, no. 12, pp. 3635-3640, 2012. DOI: 10.1109/Ted.2012.2220547.

[26] B. Hu, T. Li, J. Li, and X. Wu, "A Novel Large-Orbit Electron Gun Driving a W-Band Sixth-Harmonic Magnetron-Type Slotted Peniotron with a Permanent Magnet System," Appl. Phys. Express, vol. 6, no. 5, 2013. DOI: 10.7567/apex.6.057301.

[27] M. Glyavin, V. Manuilov, E. Taradaev, G. Sominskii, A. Fokin, and A. Sedov, "Design of a pulsed $0.5 \mathrm{THz}$ gyrotron and preliminary test of its electron gun with field emitter," Infrared Phys. and Technol., vol. 111, 2020. DOI: 10.1016/j.infrared.2020.103480.

[28] W. He, C. Donaldson, L. Zhang, K. Ronald, P. McElhinney, and A. Cross, "High power wideband gyrotron backward wave oscillator operating towards the terahertz region," Phys. Rev. Lett., vol. 110, no. 16, pp. 165101, 2013. DOI: 10.1103/PhysRevLett.110.165101.

[29] G. S. Nusinovich, O. V. Sinitsyn, L. Velikovich, M. Yeddulla, T. M. Antonsen, A. N. Vlasov, S. R. Cauffman, and K. Felch, "Startup scenarios in high-power gyrotrons," IEEE Trans. Plasma Sci., vol. 32, no. 3, pp. 841-852, 2004. DOI: 10.1109/TPS.2004.828854.

[30] J. P. Anderson, S. E. Korbly, R. J. Temkin, M. A. Shapiro, K. L. Felch, and S. Cauffman, "Design and emission uniformity studies of a 1.5-MW gyrotron electron gun," IEEE Trans. Plasma Sci., vol. 30, no. 6, pp. 2117-2123, 2002. DOI: 10.1109/Tps.2002.806639.

[31] W. B. Herrmannsfeldt, EGUN: An electron optics and gun design program, 1988.

[32] Y. Y. Lau, "Effects of cathode surface roughness on the quality of electron beams," J. Appl. Phys., vol. 61, no. 1, pp. 36-44, 1987. DOI: $10.1063 / 1.338833$

[33] C. Yuan, T. Chang, N. Chen, and Y. Yeh, "Magnetron injection gun for a broadband gyrotron backward-wave oscillator," Phys. Plasmas, vol. 16, no. 7, pp. 073109, 2009. DOI: 10.1063/1.3187903.

[34] S. E. Tsimring, "On the spread of velocities in helical electron beams," Radiophys. Quantum Electron., vol. 15, no. 8, pp. 952-961, 1972. DOI: 10.1007/BF01030951.

[35] A. W. Fliflet, A. J. Dudas, M. E. Read, and J. M. Baird, "Use of electrode synthesis technique to design MIG-type guns for high power gyrotrons," Int. J. Electron., vol. 53, no. 6, pp. 743-754, 1982. DOI: $10.1080 / 00207218208901565$. 